\section{The role of mutated SOD1 gene in synaptic stripping and MHC class I expression following nerve axotomy in ALS murine model}

\author{
Roman M. Kassa, ${ }^{1 * \S}$ Roberta \\ Bonafede, ${ }^{1 *}$ Federico Boschi, ${ }^{2}$ \\ Manuela Malatesta, ${ }^{1}$ Raffaella Mariotti ${ }^{1}$ \\ ${ }^{1}$ Department of Neurosciences, \\ Biomedicine and Movement Sciences, \\ University of Verona, Italy \\ ${ }^{2}$ Department of Computer Sciences, \\ University of Verona, Italy \\ \$Present address: Department of \\ Neurology, University of Kentucky \\ Medical Center, KY Clinic (Wing D) - \\ L445, Lexington, KY 40536-0284, USA \\ *These authors equally contributed to \\ the work
}

\section{Abstract}

Amyotrophic lateral sclerosis (ALS) is a progressive neurodegenerative disease characterized by motoneuron death. Several cellular pathways have been described to be involved in ALS pathogenesis; however, the involvement of presynaptic stripping and the related MHC class I molecules in mutant SOD1 motoneurons remains to be clarified. To this purpose, we here investigated, for the first time, the motoneurons behavior, per se and after facial axonal injury, in terms of synaptic stripping and MHC class I expression in wild-type (Wt) mice and in a murine model of ALS, the SOD1(G93A) mice, at the presymptomatic and symptomatic stage of the disease. Concerning Wt animals, we found a reduction in synaptophysin immunoreactivity and an increase of MHC class I molecules in facial motoneurons after axotomy. In uninjured motoneurons of SOD1(G93A) mice, an altered presynaptic framework was evident, and this phenomenon increased during the disease course. The alteration in the presynaptic input is related to excitatory fibers. Moreover, after injury, a further decrease of excitatory input was not associated to an upregulation of MHC class I molecules in motoneuron soma. This study demonstrates, for the first time, that the presence of mutated SOD1 protein affects the MHC class I molecules expression, altering the presynaptic input in motoneurons. Nevertheless, a positive MHC class I immunolabeling was evident in glial cells around facial injured motoneurons, underlying an involvement of these cells in synaptic stripping. This study contributes to better understand the involvement of the mutated SOD1 protein in the vulnerability of motoneurons after damage.

\section{Introduction}

Amyotrophic lateral sclerosis (ALS) is a fatal disease characterized by progressive loss of cortical, bulbar, and spinal motoneurons leading to muscle atrophy, weakness, fasciculations, spasticity, and paralysis. The incidence of ALS is 2-3/100,000 cases and the disease is fatal within 3-5 years after clinical onset due to respiratory failure. ${ }^{1}$ Several mechanisms have been implicated in the pathogenesis of ALS, including mitochondrial damage, glutamate excitotoxicity, oxidative stress, protein aggregation, neurofilament accumulation and neuroinflammation. ${ }^{2}$ However, few studies have focused on the role of the presynaptic motoneuron compartment and on the involvement of major histocompatibility complex I (MHC class I)-related molecules in the pathogenesis of ALS.

The expression of activity of MHC class I molecules, a set of classical immune molecules involved in antigen presentation, was first described in the CNS, playing a non-immune role in development and plasticity. ${ }^{3-5}$ Moreover, an unexpected role has been attributed to MHC class I molecules in the detachment of afferent synaptic boutons from injured neurons. ${ }^{6}$ Their expression has been associated with signaling mediating strength of synapse elimination, a phenomenon known as "synaptic stripping", that occurs to limit the excitatory input resulting in a positive effect on regenerative capacity after axonal injury. ${ }^{6,7}$ The MHC class I was seen upregulated in motoneurons after mice sciatic nerve transection ${ }^{8}$ in which a retrograde response at the cell body consisting of removal of synapses from the perikaryon and dendrites occurs. ${ }^{9,10}$

The synapses, and in particular, presynaptic terminals have been implicated as early sites of dysfunction following injury in the nervous system and in neurodegenerative diseases. ${ }^{11}$ Neuropathological studies on spinal cord tissue obtained from ALS patients revealed a significant reduction of presynaptic terminals at distal portions of dendrites with relative preservation of perisomatic and proximal dendrite terminals in the gray matter of the anterior horn. ${ }^{12-15}$ Other studies showed a reduction of synaptic boutons on the soma and proximal dendrites of chromatolytic or atrophic anterior horn neurons, with no changes in normalappearing neurons. ${ }^{16,17}$ However, a significant decrease in cell body area, number of synapses and total synaptic length have been reported also in normal-appearing
Correspondence: Dr. Raffaella Mariotti, Department of Neurosciences, Biomedicine and Movement Sciences, University of Verona, Strada Le Grazie 8, 37134 Verona, Italy.

Tel. +39.045.8027164 - Fax: +39.045.8027163.

E-mail: raffaella.mariotti@univr.it

Key words: Amyotrophic lateral sclerosis; SOD1(G93A) mice; motoneurons; synaptophysin; major histocompatibility complex I; facial axotomy.

Contributions: RM, principal investigator of the team, conceived the experimental study; RMK, experiments planning and performing, results analysis; $\mathrm{RB}$, contribution to experiments performing, figures planning; MM, TEM performing; FB, statistical analysis performing; RM, RB, manuscript writing and commenting.

Acknowledgments: The authors would like to acknowledge Marco Veronese for his help to plan the figures. The work was supported by AriSLA grant FGBR_7/2016 and Joint Projects grant 2015.

Conflict of interest: The authors declare that they have no conflict of interest.

Received for publication: 21 February 2018. Accepted for publication: 2 May 2018.

This work is licensed under a Creative Commons Attribution-NonCommercial 4.0 International License (CC BY-NC 4.0).

CC Copyright R.M. Kassa et al., 2018 Licensee PAGEPress, Italy

European Journal of Histochemistry 2018; 62:2904 doi:10.4081/ejh.2018.2904

lumbar motoneurons of ALS cases. ${ }^{18}$ Most of these studies assessed changes in the immunosignal of the presynaptic vesicle membrane protein, synaptophysin, in presynaptic terminals on the soma and proximal dendrites of spinal cord motoneurons and surrounding neuropil. Data from studies on a murine model of the familial form of ALS, SOD1(G93A) mice, revealed an overall decrease in synaptic boutons on lumbar motoneurons and interneurons at disease onset and during disease progression. ${ }^{19}$ Moreover, an imbalance in synaptic input to these motoneurons was found related to disease progression, with overall over inhibition. ${ }^{20}$ However, the role of the mutated SOD1 gene in MHC class I molecules expression and in the synaptic stripping after axonal injury has not been yet elucidated in facial motoneurons, that are not involved in the typical neurodegeneration of ALS.

On these bases, our study aims to evaluate, following axonal injury, the behavior of mutant SOD1 motoneurons in relation to the 
expression of MHC class I molecules. Moreover, since the expression of these molecules is associated with the synaptic stripping, the study of the presynaptic terminal was examined. To this purpose, we analyzed the reaction of facial motoneurons to axotomy in mutant SOD1(G93A) mice (mSOD1) at the presymptomatic and symptomatic stage of disease compared to wild-type (Wt) littermates. Since in this murine model the cranial motoneurons are affected later during ALS progression, the alteration of MHC class I expression and the synaptic stripping should be due to mutant SOD1. We here examined possible differences in the strength of synaptic stripping from the perikarya of facial motoneurons of presymptomatic and symptomatic mSOD1 mice compared to $\mathrm{Wt}$ littermates after unilateral facial nerve axotomy. Quantitative assessment of perisomatic synaptophysin-immunopositive boutons and size of injured facial motoneurons compared to intact ones were done in the three groups of mice. The expression of MHC class I molecules in axotomized facial motoneurons of mSOD1 mice compared to Wt mice were also studied.

\section{Materials and Methods}

\section{Animals}

Transgenic mice carrying a mutated human SOD1 gene (strain designation: B6SJL-TgN[SOD1-G93A]1Gur) were originally obtained from Jackson Laboratories (Bar Harbor, ME, USA). The animals were kept under controlled environmental parameters and veterinarian assistance. All experiments were carried out with the authorization of the Committee for Research on Laboratory Animals of the University of Verona and the Italian Ministry of Health, following the NIH Guide for the Use and Care of Laboratory Animals, in accordance with the current European Communities Council Directives (2010/63/UE), minimizing the number of animals used and avoiding their sufferance. Transgenic progeny, derived from the colony established in our animal facility, was identified by polymerase chain reaction specific for human SOD1 with G93A mutation, as outlined by the Jackson Laboratories. The time of disease onset in our colony was around 85-95 days, consistent with reports from other colonies of the same strain. ${ }^{21}$

In all mSOD1 mice, the onset and progression of disease were carefully monitored. The absence of tremors and intact extension reflex indicated the presymptomatic stage (60-65 days), while mSOD1 mice were referred to as symptomatic (95-
105 days) when they exhibited tremors, no extension reflex, hindlimb retraction and dragging hindlimbs.

\section{Tracer injection and facial nerve axotomy}

Under anesthesia (chloral hydrate; 50 $\mathrm{mg} / \mathrm{kg}$, i.p.), presymptomatic $(\mathrm{n}=4)$ and symptomatic $(n=4)$ mSOD1, and agematched Wt mice $(n=8)$ received bilateral intramuscular injections of the FluoroGold (FG; Fluorochrome LLC, Denver, CO, USA) in the mystacial pads. In particular, 5 $\mu \mathrm{L}$ of $3 \% \mathrm{FG}$ in $0.01 \mathrm{M}$ phosphate-buffered saline, $\mathrm{pH} 7.4$ (PBS) was administered in three injection sites among the five rows of mystacial vibrissae. These mice were destined for the study on changes in the expression of synaptophysin on the soma of facial motoneurons in a paradigm of slow, ongoing neurodegeneration. FG labeling is characterized by fluorescent granules in the soma sparing the nucleus and processes of neurons. ${ }^{22}$ Good FG labeling of facial motoneurons was obtained in the rat 10 days after injection in the mystacial pad, ${ }^{23}$ and in our study which utilized mice a shorter time of 7 days was selected.

A week after FG injection, under deep anaesthesia (chloral hydrate; $50 \mathrm{mg} / \mathrm{kg}$, i.p.), the left buccal and mandibular branches of the facial nerve were cut $2 \mathrm{~mm}$ from the exit of the nerve trunk from the stylomastoid foramen, and a 1-mm long segment of each branch was cut in order to impede regeneration. Unilateral facial nerve axotomy was also done in a group of mice, $(\mathrm{Wt}$ $\mathrm{n}=8$; presymptomatic $\operatorname{mSOD} 1 \mathrm{n}=4$; symptomatic mSOD1 $\mathrm{n}=4$ ) with no precedent FG injection in the whisker pads, for the study on MHC class I expression. Motor deficits indicated by paralysis of vibrissae movement and nasal tip deviation were observed on the injured side in all mice throughout the post-axotomy survival period. None of the mice exhibited impairment of the facial muscles on the intact side evaluated through the vibrissae orientation and movement. We focused 14 days (synaptophysin and MHC class I) after axotomy as this is the most informative time point after facial nerve injury in mice, as shown in our previous report. ${ }^{24}$

\section{Perfusion, tissue processing and immunohistochemistry}

Under deep anesthesia (pentobarbital, $60 \mathrm{mg} / \mathrm{kg}$, i.p.), all mice were perfused transcardially with PBS followed by a fixative solution of $4 \%$ paraformaldehyde in $0.1 \mathrm{M}$ phosphate buffer, pH 7.4 (PB). The brainstems were dissected out and put in $20 \%$ sucrose in PBS at $4^{\circ} \mathrm{C}$ overnight.

After cryoprotection, the brainstems destined for the study on synaptophysin were snap frozen in liquid nitrogen and stored at $-80^{\circ} \mathrm{C}$ until further processing. At the time of cutting, the tissue was allowed to thaw to $-20^{\circ} \mathrm{C}$ in the cryostat chamber for 30 min, embedded in optimal cutting temperature compound, cut into $20 \mu \mathrm{m}$-thick transverse cryosections and mounted on slides. The remaining brainstems, allocated to the study on MHC class I molecules, were cut into $30 \mu \mathrm{m}$-thick sections on a freezing microtome and collected in culture plates. All sections containing the facial motor nucleus were collected and divided into adjacent series of every fifth section. To visualize presynaptic terminals together with the perikarya of facial motoneurons, double immunofluorescence for synaptophysin and FG was done. For this, one series of sections were preincubated for $2 \mathrm{~h}$ in $3 \%$ bovine serum albumin (BSA) and $0.2 \%$ Triton $\mathrm{X}-100$ in $\mathrm{PB}$ and then incubated for $48 \mathrm{~h}$ with rabbit polyclonal anti-FG antibody (Chemicon, Temecula, CA, USA; $1: 2500)$ in $3 \%$ BSA and $0.5 \%$ Triton-X-100. The mouse monoclonal anti-synaptophysin antibody (Chemicon; 1:500) which labels presynaptic boutons, was added at midincubation, i.e., after $24 \mathrm{~h}$. After repeated washing, the sections were incubated for $2 \mathrm{~h}$ in $\mathrm{Cy} 3 \mathrm{~F}(\mathrm{ab})_{2}$ fragment goat anti-rabbit IgGs (Jackson ImmunoResearch Laboratories, West Grove, PA, USA; 1:100) and fluorescein horse anti-mouse (Vector, Burlingame, CA, USA; 1:200) in 1\% BSA and $0.2 \%$ Triton $\mathrm{X}-100$ in PB. After rinsing, sections were air-dried and coverslipped with an anti-fading mounting medium (Dako, Glostrup, Denmark).

Single immunohistochemistry for light microscopy was, on the other hand, performed for MHC class I molecules. Freefloating sections were incubated for $15 \mathrm{~min}$ in $0.3 \% \mathrm{H}_{2} \mathrm{O}_{2}$ to quench endogenous peroxidase and preincubated for $2 \mathrm{~h}$ in $3 \%$ BSA and $0.2 \%$ Triton $\mathrm{X}-100$ in PBS. They were then incubated overnight in monoclonal mouse anti-mouse $\mathrm{H}-2 \mathrm{~K}^{\mathrm{b}} / \mathrm{H}-2 \mathrm{D}^{\mathrm{b}}$ antibody (BD Pharmingen, Erembodegem, Belgium; $1: 200)$, which recognizes the classical $\mathrm{H}$ $2 \mathrm{~K}^{\mathrm{b}}$ and $\mathrm{H}-2 \mathrm{D}^{\mathrm{b}} \mathrm{MHC}$ class I alloantigens, in $3 \%$ BSA in PBS. After rinsing, the sections were incubated for $2 \mathrm{~h}$ in biotinylated horse anti-mouse IgGs (Vector; 1:200) in 1\% BSA in PBS. The reaction was developed with the avidin-biotin-peroxidase kit (ABC kit; Vector) using 3-3'-diaminobenzidine as chromogen. No immunopositivity was seen in control sections processed by eliminating primary antibodies. After mounting on slides, the sections were dehydrated through increasing grades of ethanol, cleared in xylene, and coverslipped with Entellan (Merck, Darmstadt, Germany). 


\section{Data analysis}

Sections processed for immunoperoxidase were observed under the microscope under bright-field illumination.

Immunofluorescence was analyzed with a Zeiss LSM 510 confocal laser scanning microscope, in a dual-channel acquisition setup, using $488 \mathrm{~nm}$ and $543 \mathrm{~nm}$ excitation beams. Facial motoneurons were identified by their FG-immunolabelling. Images of randomly-selected facial motoneurons from the intact and axotomized sides $(n=10 /$ side from two sections/animal at similar levels of the facial motor nucleus) were collected. Under identical conditions, including constant pinhole size for both excitation beams in a merged mode, Z-axis image stacks of $1024 \times 1024$ pixels/image were acquired with a $63 \mathrm{x}$ water immersion objective (1.40 numerical aperture) and 1.2x scan zoom. The optimal distance between stacks was automatically adjusted to fit the axial sampling. Care was taken in setting confocal laser scanning parameters to allow optimal visualization of punctuating staining on neural cell membranes with clear contours of immunopositive puncta and good resolution among contiguous ones. For quantitative analysis of the number of presynaptic boutons on the perikarya of sampled facial motoneurons and measurement of the perimeter of respective neurons, the software Zeiss LSM Image Browser was used. Immunolabelled presynaptic boutons were considered to be in juxtaposition to FG-immunolabelled facial motoneurons when no space was visible between the bouton and the neuronal cell membrane in an optical section. On Zstack images consisting of the whole 3dimensional extent of sampled neurons, a single neuron was zoomed in on and perpendicular lines dividing the neuron into quadrants that were drawn using the 'Overlay' function. Focusing on one quadrant at a time on the first to the last optical section containing a selected neuron, the number of boutons touching the cell surface were counted manually after comparing with adjacent optical sections to avoid double counting. Minor adjustments in the contrast of images were made whenever necessary to have a clearer separation between puncta. The perimeter of sampled facial motoneurons was measured by tracing the outline by using the 'Overlay' function. Synaptic bouton density over the entire motoneuron cell bodies was computed as a ratio of the total number of synaptic boutons/neuron to the perimeter.

\section{Evaluation of synapses at transmis- sion electron microscopy}

Under deep anesthesia, presymptomatic $(n=4)$ and symptomatic $(n=4)$ SOD1-mutant mice, and age-matched $\mathrm{Wt}(\mathrm{n}=8)$ mice were perfused transcardially with PBS followed by a fixative solution of $2.5 \%$ glutaraldehyde and $2 \%$ paraformaldehyde in $0.1 \mathrm{M}$ $\mathrm{PB}, \mathrm{pH}$ 7.4. The brainstem regions containing the facial motor nucleus were dissected out and immersed in the same fixative solution for $3 \mathrm{~h}$ at $4{ }^{\circ} \mathrm{C}$, post-fixed with $1 \% \mathrm{OsO}_{4}$ at $4^{\circ} \mathrm{C}$ for $1 \mathrm{~h}$, dehydrated with acetone and embedded in Epon 812.

Ultrathin sections $(70-90 \mathrm{~nm})$ were placed on copper grids and observed in a Philips Morgagni transmission electron microscopy apparatus equipped with a Megaview II camera for digital image acquisition and a computerized image analysis system (AnalySIS Image processing, Soft Imaging System GmbH, Muenster, Germany). For morphometrical analyses of synapses, 5 motoneurons cut in the nuclear plane were considered in each facial motor nucleus per animal. The cellular perimeter and the length of the synaptic terminals opposing to the motoneuron somata were measured; then, the number of synapses per $100 \mu \mathrm{m}$ cell membrane and the percentage of cell membrane covered by the synaptic terminals were calculated. The terminals were classified into asymmetric synapses with spherical (S) synaptic vesicles, considered to be excitatory in nature, and symmetric synapses with flattened (F) synaptic vesicles, associated with inhibitory neurotransmitter. ${ }^{25}$

\section{Statistical evaluation}

The between-group difference in the total number of synaptic boutons (total bouton count/side/neuron/group) and synaptic density (synaptic density/side/neuron, i.e., ratio of total bouton count/side/neuron to neuron perimeter/group) and the numbers of type $\mathrm{S}$ and $\mathrm{F}$ fibers on $100 \mu \mathrm{m}$ cell perimeter were analyzed with a 2-way analysis of variance and Bonferroni posthoc test. Statistical analysis was performed using a routine written in Matlab 7.1. Significance was set at $\mathrm{P}<0.05$.

\section{Results}

Bright retrograde fluorescent tracer FG-labeling of facial motoneurons on the intact and axotomized sides was obtained in all three groups of animals: Wt, presymptomatic and symptomatic SOD1(G93A) mice.

\section{Synaptophysin immunoreactivity}

Synaptophysin is the most commonly used immunohistological marker for the identification and quantitative assessment of synapses. ${ }^{26}$ Synaptophysin immunostaining was seen as fine granules on the surface of the soma of neurons and throughout the neuropil, as previously described. ${ }^{27}$
In the facial motor nucleus, attenuated synaptophysin immunosignal was detected on the side of injury compared to the contralateral one in all three groups of animals (Figure 1). Upon detailed examination, in Wt mice, areas devoid of synaptophysinimmunopositive boutons were detected on the perikarya of axotomized facial motoneurons, identified by FG-immunolabelling, compared to those from the intact side (Figure 1A,D). Quantitative evaluation of the synaptophysin-immunoreactive presynaptic terminals revealed a reduction in the number of synapses on Wt axotomized facial motoneurons compared to uninjured ones $(\mathrm{P}=0.00001$; Figure 2A). Analysis of the synaptic density, on the other hand, showed a significant decrease in Wt facial motoneurons after axotomy ( $\mathrm{P}=0.00001$; Figure 2B). Interestingly, in presymptomatic SOD1(G93A) mice, segments of the surface of the soma lacking synaptic boutons were detected on uninjured facial motoneurons compared to intact cells from Wt mice (Figure 1 A,B). Following axotomy, a detachment of presynaptic terminals in injured presymptomatic SOD1(G93A) facial motoneurons were found compared to the intact side (Figure 1 $\mathrm{B}, \mathrm{E})$. Intriguingly, the number and density of synapses on intact facial motoneurons from presymptomatic mSOD1 mice were similar to that in axotomized Wt ones, while they are different to that of uninjured $\mathrm{Wt}$ (mean number $\mathrm{P}=0.0020$; mean synaptic density $\mathrm{P}=0.0013$. Figure $2 \mathrm{~A}, \mathrm{~B})$. Moreover, a decrease in the number of synaptic boutons $(\mathrm{P}=0.00001)$ and in the synaptic density $(\mathrm{P}=0.00001)$ (Figure $2 \mathrm{~A}, \mathrm{~B})$ was found in injured presymptomatic SOD1(G93A) facial motoneurons compared to the intact side. In addition, the axotomized presymptomatic mSOD1 motoneurons had less number $(\mathrm{P}=0.0007)$ and density $(\mathrm{P}=0.0018)$ of synapses compared to axotomized $\mathrm{Wt}$ cells (Figure 2 A,B).The uninjured facial motoneurons from symptomatic mSOD1 mice showed stretches of the surface of the soma compared to uninjured Wt neurons (Figure $1 \mathrm{~A}, \mathrm{C}$ ). Moreover, a reduction of synaptic boutons after axotomy was detected, similar to $\mathrm{Wt}$ and presymptomatic SOD1(G93A) mice (Figure 1 C,F). Concerning the uninjured facial motoneurons from symptomatic mSOD1 mice, there was a tendency towards a reduction in the number of synapses compared to uninjured Wt (Figure 2A). The synapse loss from uninjured facial motoneurons from symptomatic mSOD1 mice was not different from that found in presymptomatic mice and paralleled the synaptic stripping in axotomized Wt motoneurons (Figure 2A). Synaptic density, on the other hand, was significantly reduced in intact facial motoneurons from 
symptomatic mSOD1 mice compared to unlesioned Wt $(\mathrm{P}=0.00001$; Figure 2B). As in both $\mathrm{Wt}$ and presymptomatic SOD1(G93A) mice, following axotomy, in symptomatic mSOD1 mice, a significant loss of synaptic boutons $(\mathrm{P}=0.00001)$ and decreased synaptic density $(\mathrm{P}=0.00001)$ was found on injured motoneurons compared to intact ones (Figure 2 A,B). Furthermore, synaptic density on axo- tomized facial motoneurons from symptomatic mSOD1 mice was significantly reduced compared to axotomized $\mathrm{Wt}$ motoneurons ( $\mathrm{P}=0.00001$; Figure $2 \mathrm{~B})$.
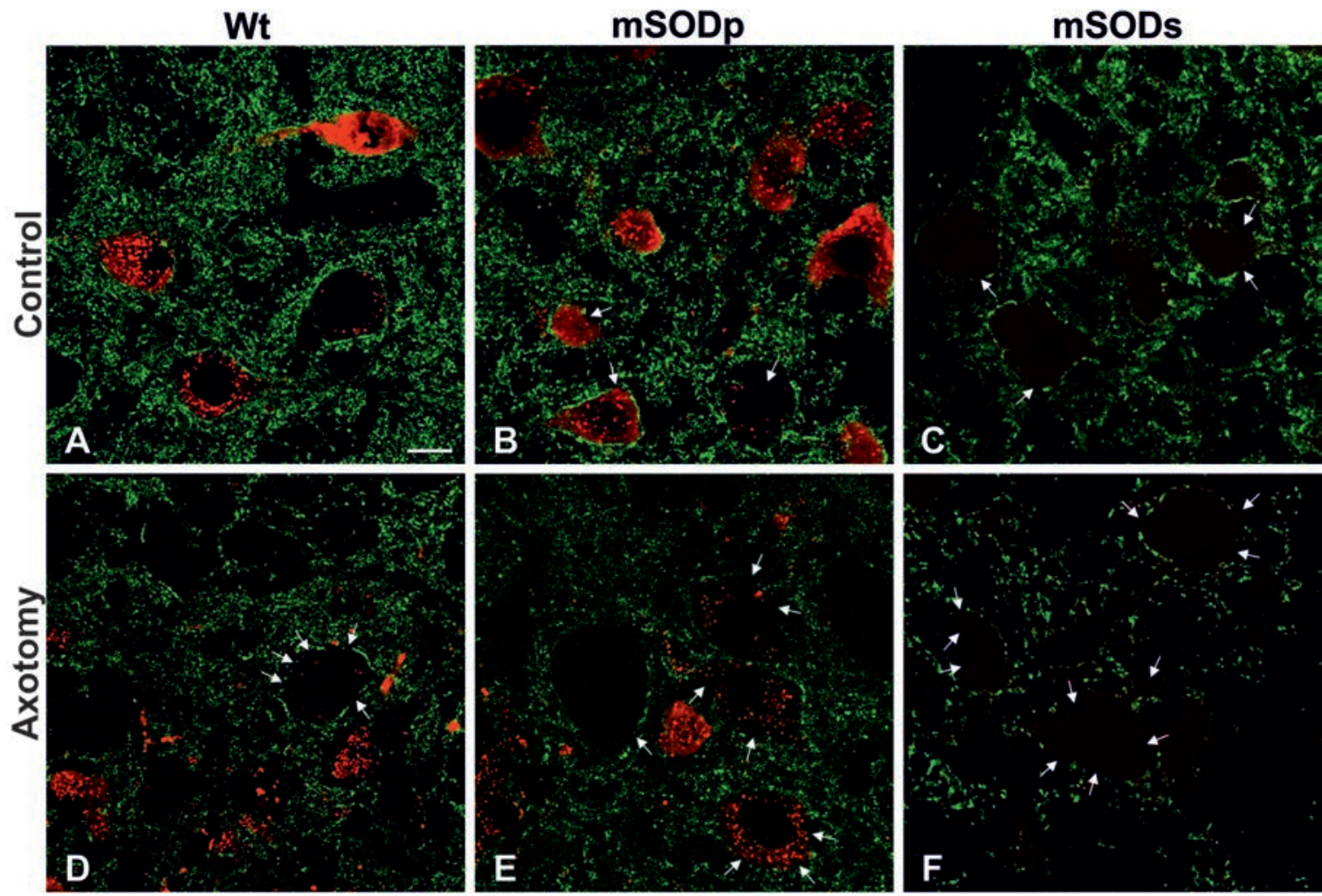

Figure 1. Synaptophysin immunofluorescent staining. Double immunofluorescent staining of the facial motor nucleus for synaptophysin (green) and FluoroGold (red) 14 days after facial nerve resection. Arrows indicate areas devoid of synaptophysin-immunoreactive boutons. Axotomy, axotomized side; Control, intact side; Wt, wild-type; mSODp, presymptomatic SOD1(G93A); mSODs, symptomatic SOD1(G93A). Scale bar: $20 \mu \mathrm{m}$.

A

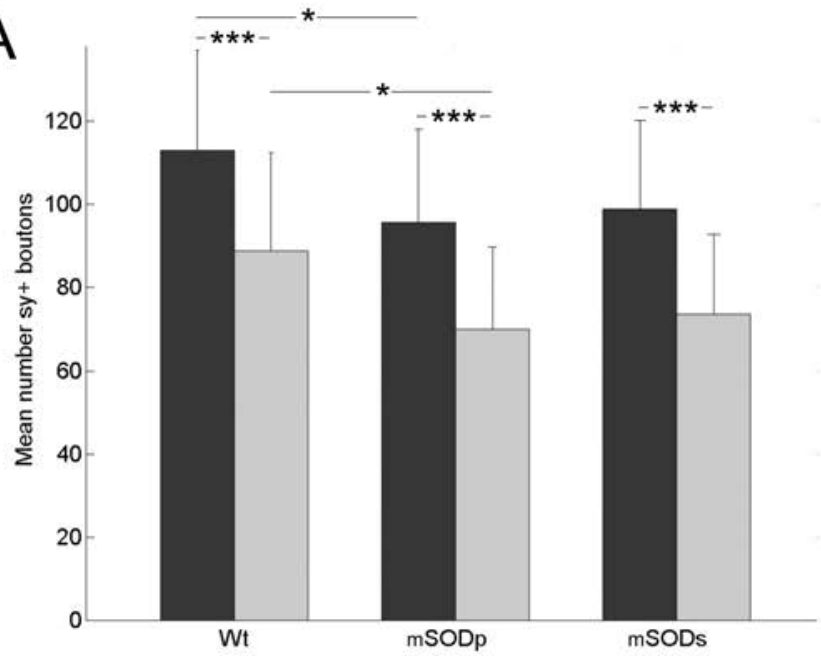

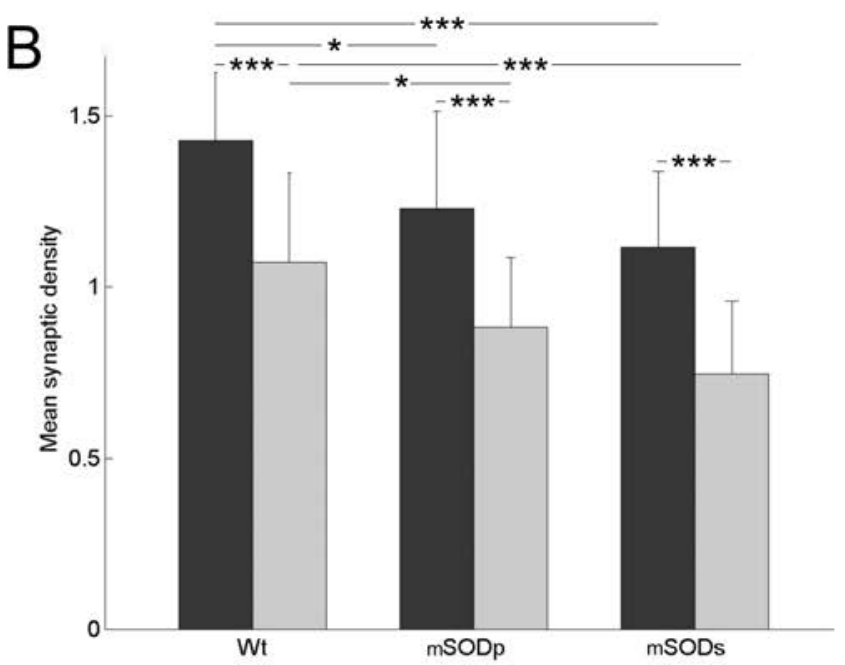

Figure 2. Synaptic boutons and density. Graphs showing the mean values and respective standard deviations for synaptic boutons (A) and synaptic density (B) of wild-type (Wt), presymptomatic SOD1(G93A) (mSODp) and symptomatic SOD1(G93A) (mSODs) mice. The black columns indicate the intact side, grey columns indicate the axotomized side. In the figure are shown the statistically significant differences $\left({ }^{*} \mathrm{P}<0.0033 ;{ }^{* * *} \mathrm{P}<0.000066\right)$. 


\section{Morphological examination of synapsis}

The ultrastructural examination of synapses confirms the synaptophysin immunolabeling results, in which a reduction of the presynaptic boutons between $\mathrm{Wt}$ and SOD1(G93A) mice was evaluated. In
Figure 3 are reported the representative TEM images of excitatory and inhibitory synapsis. It is important to underline that the presynaptic buttons reduction is particularly related to excitatory fibers (types S) instead to inhibitory one (type F), as shown in Figure 4A.
The excitatory fibers input on unlesioned facial motoneurons of presymptomatic mutant SOD1 mice is already compromised compared to $\mathrm{Wt}$, showing a significant statistical reduction $(\mathrm{P}=0.00001)$ of the type $\mathrm{S}$ fiber number (11.06 and 20.77 number of type $\mathrm{S} / 100 \mu \mathrm{m}$ cell perimeter in
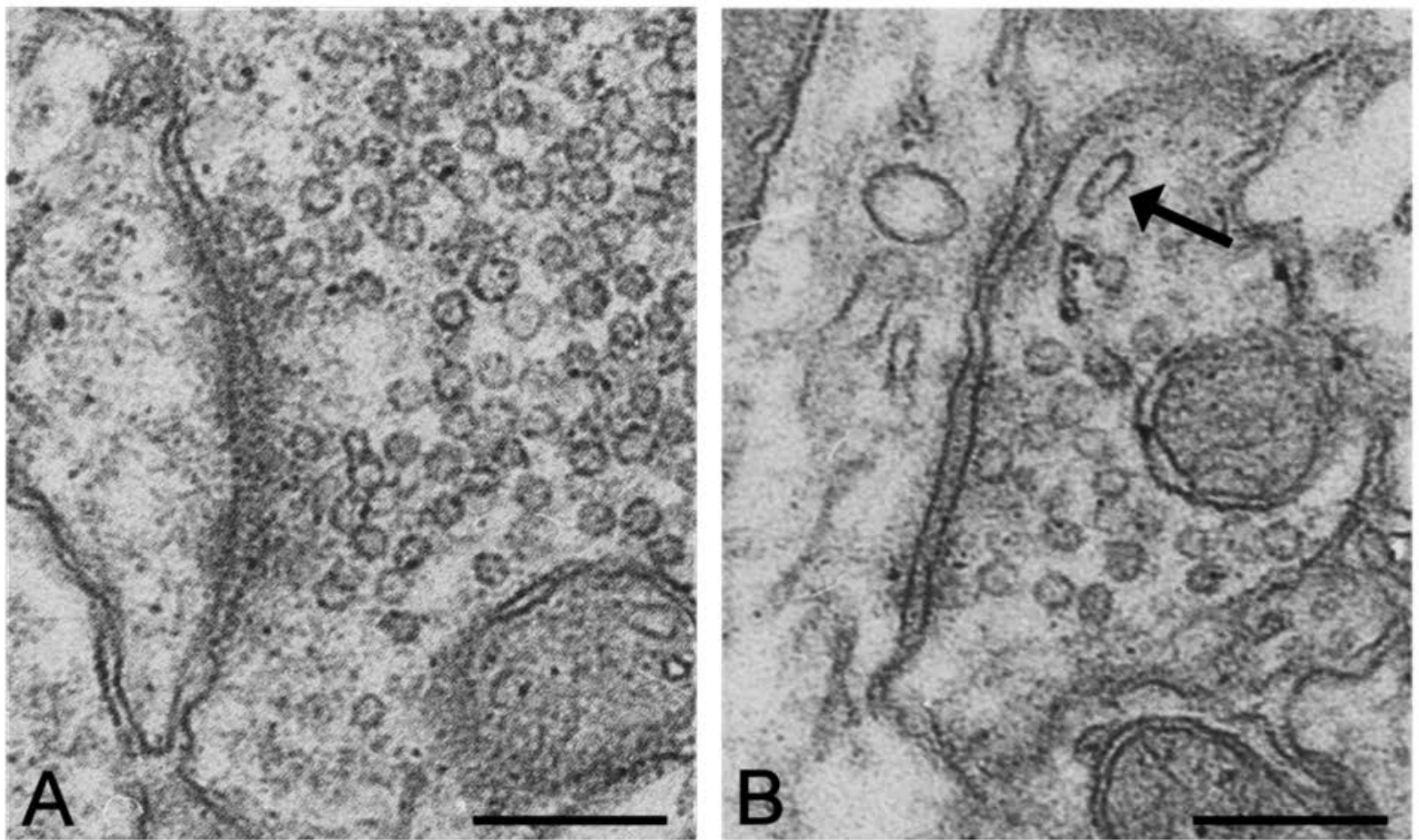

Figure 3. Synapses ultrastructure. Representative transmission electron micrographs of an asymmetric (excitatory) synapsis with spherical vesicles (A), and a symmetric (inhibitory) synapsis showing typically flattened vesicles (arrow) (B). Scale bars: $200 \mathrm{~nm}$.
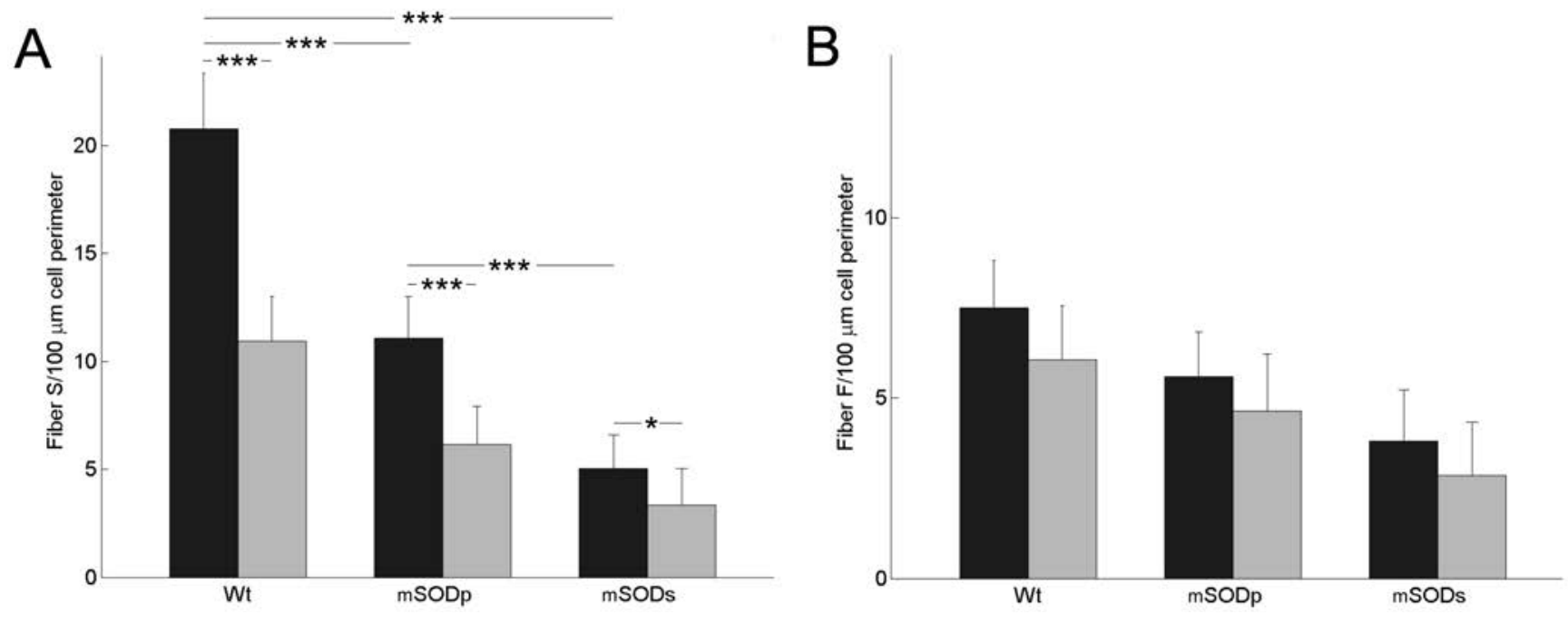

Figure 4. Excitatory and inhibitory fibers. Graphs showing the morphological examination of synapsis of wild-type (Wt), presymptomatic SOD1(G93A) (mSODp) and symptomatic SOD1(G93A) (mSODs) mice. In A are reported the results concerning the number of excitatory fibers (type $S$ ) $/ 100 \mu \mathrm{m}$ of cell perimeter; in $\mathrm{B}$ are reported the results concerning the number of inhibitory fibers (type $\mathrm{F}$ )/100 $\mu \mathrm{m}$ of cell perimeter. The black columns indicate the intact side, grey columns indicate the axotomized side. In the figure are shown some of the statistically significant differences $\left({ }^{*} \mathrm{P}<0.0033 ;{ }^{* * *} \mathrm{P}<0.000066\right)$. 
presymptomatic $\mathrm{mSOD} 1$ and $\mathrm{Wt}$ mice respectively; Figure 4A). These data indicate that the excitatory input is already altered at the presymptomatic stage of the disease. Moreover, during the disease course, a further reduction of excitatory fibers input occurs in intact facial motoneurons, showing a significant statistical difference compared to Wt $(\mathrm{P}=0.00001)$ and presymptomatic mSOD1 mice $(\mathrm{P}=0.00001$; Figure 4A). After axotomy, in Wt mice, a $50 \%$ of excitatory fiber reduction was found (10.94 of S fibers on $100 \mu \mathrm{m}$ cell perimeter) and this trend was detected also in presymptomatic and symptomatic mSOD1 mice (6.17 and 3.35 of $\mathrm{S}$ fibers on cell perimeter respectively; Figure 4B). Concerning type F fibers, no statistical significance was observed comparing the axotomized and unlesioned side of three groups of animals (Figure 4B), indicating a selective loss of type $\mathrm{S}$ fibers after injury. Moreover, no statistical difference was observed between unlesioned $\mathrm{Wt}$ and presymptomatic mSOD1 mice. This indicates that mSOD1 facial motoneurons injured behave as $\mathrm{Wt}$, modulating the excitatory input and not inhibitory one.

\section{MHC class I immunoreactivity}

In Wt mice, MHC class I molecules expression was detected in uninjured facial motoneurons, evidenced by faint immunolabelling of the rims of the cytoplasm of intact one (Figure $5 \mathrm{~A}, \mathrm{~B}$ ). In axotomized facial motoneurons of Wt mice, the MHC I immunolabelling pattern show an intense, homogenous and diffuse staining of the cell body (Figure $5 \mathrm{C}, \mathrm{D})$. The expression of MHC class I molecules was detected as faint immunolabelling also in the uninjured facial motoneurons of mSOD1, as in $\mathrm{Wt}$ mice. After axotomy, in presymptomatic mSOD1 mice, MHC class I immunolabelling was seen inside motoneurons with a diffuse distribution over the cell body that seems to decrease in intensity compared to Wt (Figure 5 C,D-E,F). In symptomatic mSOD1 mice, it was evident an upregulation in the expression of MHC class I protein on the lesioned side compared to the intact one (data not shown) and compared to $\mathrm{Wt}$ and presymptomatic mSOD1 mice showed an immunolabelling limited to markedly activated microglia, recognized by morphology (Figure 5).

\section{Discussion}

Murine SOD1(G93A) facial motoneurons represent a model to study the involvement of the mutant SOD1 gene in ALS and to examine their dieback response after axotomy, since they do not die during the disease course, ${ }^{28}$ differently to human ALS. ${ }^{2}$

Our data in SOD1(G93A) facial motoneurons show a diminished number of presynaptic terminals compared to $\mathrm{Wt}$. The number and density of presynaptic terminals in intact facial motoneurons of mSOD1 mice were decreased compared to $\mathrm{Wt}$, underlining that mutated SOD1 facial motoneurons show a compromised presynaptic input. Interestingly, loss of excitatory input and unchanged inhibitory input, resulting in over-inhibition rather than overexcitation, was reported in lumbar motoneurons from symptomatic SOD1(G93A) mice..$^{20}$ In accordance to this, we demonstrate that the altered presynaptic input in facial motoneurons regards the excitatory fibers instead to inhibitory ones. The decrease of the number and density of presynaptic terminals in mSOD1 mice were more evident in symptomatic compared to the presymptomatic animals, indicating that, despite this motoneurons do not die, their impairment increase during the disease course. Since it is reported that excitatory synaptic stripping occurs in motoneurons after injury as a defense mechanism, ${ }^{6}$ we think that the excitatory fibers reduction observed in our study serves to counteract the excitotoxic influence on uninjured mutated SOD1 motoneurons, indicating that the SOD1 mutation di per se induces a pathological motoneuron condition.

Following axotomy, the number of detached synapses did not seem to vary with progression of the disease in this ALS murine model. However, a reduction in synapse density was found to correlate with the progression of the disease and the axotomized mSOD1 facial motoneurons had a significant decrease in synaptic density compared to injured Wt counterparts.

Early and gradual loss of synapses is a pathological feature common to ALS disease. ${ }^{29}$ This process has been extensively studied in the spinal motoneurons, directly affected by the neurodegeneration, during the disease course, allowing to study the synaptic stripping in degenerating motoneu-
Wt

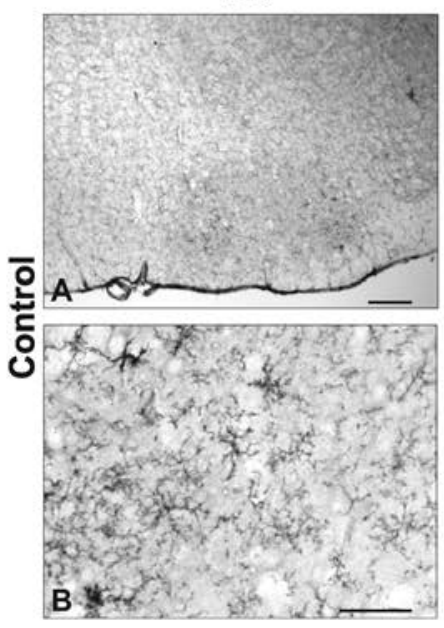

Wt

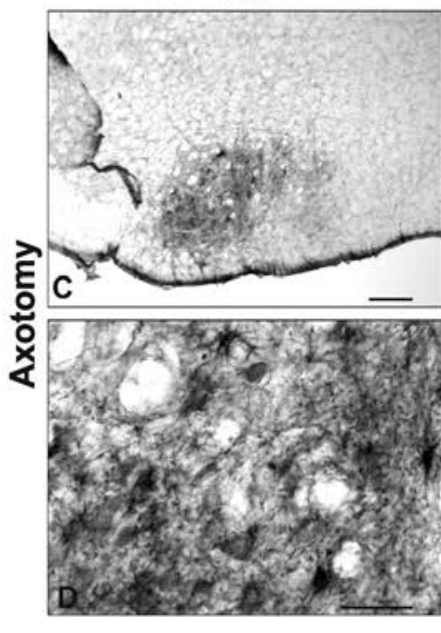

mSODp

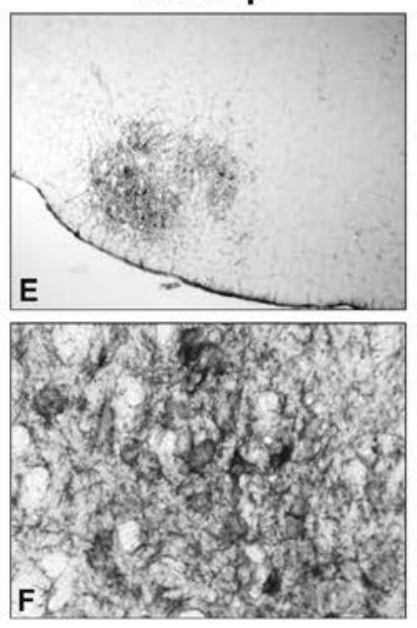

mSODs

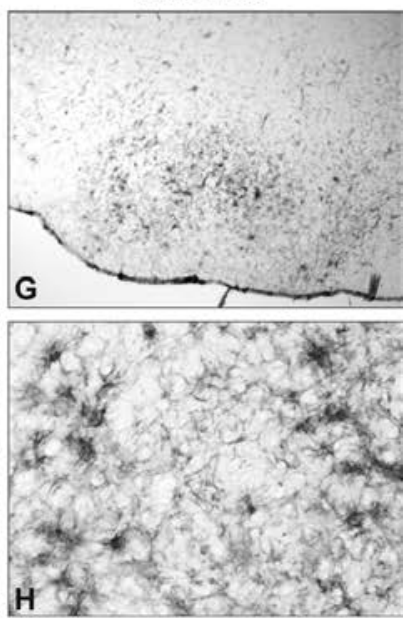

Figure 5. MHC class I molecules immunolabeling. In Wt mice, note a faint immunolabelling of MHC class I molecules expression in uninjured facial motoneurons (A,B). MHC class I immunolabeling in the axotomized facial motor nucleus of wild-type (Wt) (C,D), presymptomatic SOD1(G93A) (mSODp) (E,F) and symptomatic SOD1(G93A) (mSODs) (G,H) mice 14 days after axotomy. Scale bars: A,C,E,G) $200 \mu \mathrm{m} ; \mathrm{B}, \mathrm{D}, \mathrm{F}, \mathrm{H}): 50 \mu \mathrm{m}$. 
rons. The decrease in the number and density of synaptophysin-immunoreactive boutons from the soma and proximal dendrites of lumbar motoneurons and interneurons of SOD1(G93A) mice was found at disease onset, with ongoing loss through disease progression. ${ }^{19}$ However, this study does not allow to correlate the synaptic stripping to the SOD1 mutation. Our findings on facial motoneurons of SOD1(G93A) mice showed a reduced number of presynaptic terminals compared to the Wt even before any signs of disease, indicating a selective involvement of the mutated SOD1 gene in the vulnerability of the motoneurons after damage.

Interesting differences between $\mathrm{Wt}$ and transgenic mice in the upregulation of the expression of MHC class I molecules in facial motoneurons following axotomy were also detected. Comparing SOD1(G93A) mice to the $\mathrm{Wt}$, the expression of MHC class I molecules decrease during the progression of the disease. MHC class I molecules, classically thought to mediate exclusively immune function, have been implicated in neuronal signaling in vivo and activity-dependent changes in synaptic connectivity. ${ }^{5}$ Moreover, it has been reported that MHC class I protein can be located at both pre- and post-synaptic level in the healthy $\mathrm{CNS}^{30}$ and its expression increased after injury, playing a potential role in reparative processes and mediating synaptic stripping. In relation to this, an injury cell response is represented by soma presynaptic terminal elimination, that primarily involves excitatory input instead of inhibitory fibers, ${ }^{6}$ that seem to be preserved. This process leads to a surplus of inhibitory influence on injured neurons and may be of benefit by directing the cellular mechanism towards repair rather than information processing. ${ }^{31}$ This "neuroprotective" response to peripheral nerve lesion may thus be impaired in mSOD1 mice. Moreover, impaired MHC class I signaling has been associated with an impediment in regenerative capacity. ${ }^{32,33}$ Studies in six weeks old dystrophic MDX mice showed a reduced synaptic activity in intact lumbar motoneurons, more intense synaptic detachment and lower MHC I upregulation compared to the Wt following sciatic nerve transection. ${ }^{34}$ Intriguingly, inducible nitric oxide synthase knockout mice showed increased synaptic stripping and decreased MHC class I expression around axotomized lumbar motoneurons compared to the $\mathrm{Wt}^{35}$ Impairment in the activation of the nitrergic and purinergic systems in facial motoneurons in response to facial nerve axotomy has been previously described in this mouse model of familial ALS. ${ }^{24,36}$

The data from the present study show that mSOD1 expression alters motoneurons functionality, that attempt to counteract the intracellular damage altering the excitatory presynaptic input. Despite MHC class I molecules seem to have an effect on synaptic stripping inducing regeneration, in SOD1(G93A) motoneurons after injury we did not find an upregulation in MHC class I molecules expression compared to $\mathrm{Wt}$, although an excitatory synaptic stripping occurs. The presence of mSOD1 protein could interfere with MHC class I expression: it is known that the ubiquitin-proteasome system, whose functionality is altered in ALS, is involved in the generation of $\mathrm{MCH}$ class I molecules. ${ }^{37}$ Moreover, the crosstalk between damaged motoneurons and glia could induce the microglial cells to express MHC I in order to induce synaptic stripping. It was already hypothesized an involvement of microglial cells in synaptic stripping after facial nerve transection. ${ }^{38}$ In fact, we found, in SOD1(G93A) facial nucleus, an MHC immunolabelling limited to activated microglia, which underlies a potential role of microglial cells in presynaptic removal. Since in motoneurons of SOD1(G93A) mice there is an impairment of $\mathrm{MHC}$ production, we believe that the synaptic rearrangement could be due to MHC expressed by microglia. The role of mutated microglia in the ALS disease is controversial and there is evidence that its beneficial/detrimental role depends on its different phenotypes (M1 and M2) during the disease progression. ${ }^{39,40}$ In our study, the MHC I expression in microglial cells could indicate its protective role in the non-late stage of the disease of mice, trying to rescue axotomized motoneurons from death.

In summary, our data provide new evidence of the importance of MHC class I in synaptic stripping, and the better understanding of the complex scenario that occurs in presence of mutated SOD1 protein could help to develop new strategies to rescue ALS motoneurons.

\section{References}

1. Costa J, de Carvalho M. Emerging molecular biomarker targets for amyotrophic lateral sclerosis. Clinica Chimica Acta 2016;30010-19.

2. Bonafede R, Mariotti R. ALS pathogenesis and therapeutic approaches: the role of mesenchymal stem cells and extracellular vesicles. Front Cell Neurosci 2017;11:80.

3. Boulanger LM. Immune proteins in brain development and synaptic plasticity. Neuron 2009;64:93-109.

4. Shatz CJ. MHC class I: an unexpected role in neuronal plasticity. Neuron 2009;64:40-5.
5. Elmer BM, McAllister AK. Major histocompatibility complex class I proteins in brain development and plasticity. Trends Neurosci 2012;35:660-70.

6. Spejo AB, Oliveira AL. Synaptic rearrangement following axonal injury: Old and new players. Neuropharmacology 2015;96:113-23.

7. Cebrián C, Loike JD, Sulzer D. Neuronal MHC-I expression and its implications in synaptic function, axonal regeneration and Parkinson's and other brain diseases. Front Neuroanat 2014;8:114.

8. Victório SC, Cartarozzi LP, Hell RC, Oliveira AL. Decreased MHC I expression in IFN $\gamma$ mutant mice alters synaptic elimination in the spinal cord after peripheral injury. J Neuroinflammation 2012;7:9-88.

9. Blinzinger K, Kreutzberg G. Displacement of synaptic terminals from regenerating motoneurons by microglial cells. Z. Zellforsch Mikrosk Anat 1968;85:145-57.

10. Moran LB, Graeber MB. The facial nerve axotomy model. Brain Res Rev 2004:44:154-78.

11. Gould TW, Oppenheim RW. Synaptic dysfunction in disease and following injury in the developing and adult nervous system: Caveats in the choice of therapeutic intervention. Neurosci Biobehav Rev 2007;31:1073-87.

12. Matsumoto S, Goto S, Kusaka H, Ito H. Imai T. Synaptic pathology of spinal anterior horn cells in amyotrophic lateral sclerosis: an immunohistochemical study. J Neurol Sci 1994;125:180-5.

13. Ince PG, Slade J, Chinnery RM, McKenzie J, Royston C, Roberts GW, et al. Quantitative study of synaptophysin immunoreactivity of cerebral cortex and spinal cord in motor neuron disease. J Neuropathol Exp Neurol 1995;54:673-9.

14. Ikemoto A, Hirano A. Comparative immunohistochemical study on synaptophysin expression in the anterior horn of post-poliomyelitis and sporadic amyotrophic lateral sclerosis. Acta Neuropathol 1996;92:473-8.

15. Ikemoto A, Nakamura S, Akiguchi I, Hirano A. Differential expression between synaptic vesicle proteins and presynaptic plasma membrane proteins in the anterior horn of amyotrophic lateral sclerosis. Acta Neuropathol 2002;103:179-87.

16. Sasaki S, Maruyama S. Decreased synaptophysin immunoreactivity of the anterior horns in motor neuron disease. Acta Neuropathol 1994;87:125-28.

17. Cruz-Sánchez FF, Moral A, Rossi ML, Quintó L, Castejòn C, Tolosa E, et al. 
Synaptophysin in spinal anterior horn in aging and ALS: an immunohistological study. J Neural Transm 1996;103:131729.

18. Sasaki S, Maruyama S. Synapse loss in anterior horn neurons in amyotrophic lateral sclerosis. Acta Neuropathol 1994;88:222-7.

19. Zang DW, Lopes EC, Cheema SS. Loss of synaptophysin-positive boutons on lumbar motor neurons innervating the medial gastrocnemius muscle of the SOD1G93A G1H transgenic mouse model of ALS. J Neurosci Res 2005;79: 694-9.

20. Schütz B. Imbalanced excitatory to inhibitory synaptic input precedes motor neuron degeneration in an animal model of amyotrophic lateral sclerosis. Neurobiol Dis 2005;20:131-40.

21. Turner BJ, Talbot K. Transgenics, toxicity and therapeutics in rodent models of mutant SOD1-mediated familial ALS. Progr Neurobiol 2008;85:94-134.

22. Schmued LC, Fallon JH. Fluoro-Gold: a new fluorescent retrograde axonal tracer with numerous unique properties. Brain Res 1986;377:147-54.

23. Popratiloff AS, Neiss WF, Skouras E, Streppel M, Guntinas-Lichius O, Angelov DN. Evaluation of muscle reinnervation employing pre- and postaxotomy injections of fluorescent retrograde tracers. Brain Res Bull 2001;54: 115-23.

24. Mariotti R, Cristino L, Bressan C, Boscolo S, Bentivoglio M. Altered reaction of facial motoneurons to axonal damage in the presymptomatic phase of murine model of familial amyotrophic lateral sclerosis. Neuroscience 2002;
115:331-35.

25. Peters A, Palay SA, Webster F. Fine structure of the nervous system: neurons and their supporting cells. Oxford University Press; New York; 1991.

26. Valtorta F, Pennuto M, Bonanomi D, Benfenati F. Synaptophysin: leading actor or walk-on role in synaptic vesicle exocytosis? Bioessays 2004;26:445-53.

27. Wiedenmann B, Franke WW. Identification and localization of synaptophysin, an integral membrane glycoprotein of Mr 38,000 characteristic of presynaptic vesicles. Cell 1985;41: 1017-28.

28. Mariotti R, Bentivoglio M. Activation and response to axotomy of microglia in the facial motor nuclei of G93A superoxide dismutase transgenic mice. Neurosci Lett 2000;285:87-90.

29. Schafer DP, Stevens B. Synapse elimination during development and disease: immune molecules take centre stage. Biochem Soc Trans 2010;38:476-81.

30. Needleman LA, Liu XB, El-Sabeawy F, Jones EG, McAllister AK. MHC class I molecules are present both pre- and postsynaptically in the visual cortex during postnatal development and in adulthood. Proc Natl Acad Sci USA 2010;107:16999-7004.

31. Thams S, Oliveira A, Cullheim S. MHC class I expression and synaptic plasticity after nerve lesion. Brain Res Rev 2008;57:265-9.

32. Oliveira AL, Thams S, Lidman O, Piehl F, Hökfelt T, Kärre K, et al. A role for MHC class I molecules in synaptic plasticity and regeneration of neurons after axotomy. Proc Natl Acad Sci USA 2004;101:17843-8.

33. Sabha MJr, Emirandetti A, Cullheim S,
De Oliveira AL. MHC I expression and synaptic plasticity in different mice strains after axotomy. Synapse 2008;62:137-48.

34. Simões GF, Oliveira ALR. Alpha motoneurone input changes in dystrophic MDX mice after sciatic nerve transection. Neuropathol Appl Neurobiol 2010;36:55-70.

35. Emirandetti A, Simões GF, Zanon RG, Oliveira ALR. Spinal motoneuron synaptic plasticity after axotomy in the absence of inducible nitric oxide synthase. J Neuroinflammation 2010;24:731.

36. Kassa RM, Bentivoglio M, Mariotti R. Changes in the expression of $\mathrm{P} 2 \mathrm{X}_{1}$ and $\mathrm{P} 2 \mathrm{X}_{2}$ purinergic receptors in facial motoneurons after nerve lesions in rodents and correlation with motoneuron degeneration. Neurobiol Dis 2007;25:121-33.

37. Bendotti C, Marino M, Cheroni C, Fontana E, Crippa V, Poletti A, et al. Dysfunction of constitutive and inducible ubiquitin-proteasome system in amyotrophic lateral sclerosis: implication for protein aggregation and immune response. Prog Neurobiol 2012;97:101-26.

38. Perry VH, O'Connor V. The role of microglia in synaptic stripping and synaptic degeneration: a revised perspective. ASN Neuro 2010;2:e00047.

39. Brites D, Vaz AR. Microglia centered pathogenesis in ALS: insights in cell interconnectivity. Front Cell Neurosci 2014;8:117.

40. Komine O, Yamanaka K. Neuroinflammation in motor neuron disease. Nagoya J Med Sci 2015;77: 537-49. 